Acta Crystallographica Section F

Structural Biology

and Crystallization

Communications

ISSN 1744-3091

Debora L. Makino, Agnes H. Henschen-Edman and Alexander McPherson*

Molecular Biology and Biochemistry, University of California, Irvine, 560 Steinhaus Hall, Irvine, CA 92697-3900, USA

Correspondence e-mail: amcphers@uci.edu

Received 10 September 2004

Accepted 5 November 2004

Online 2 December 2004

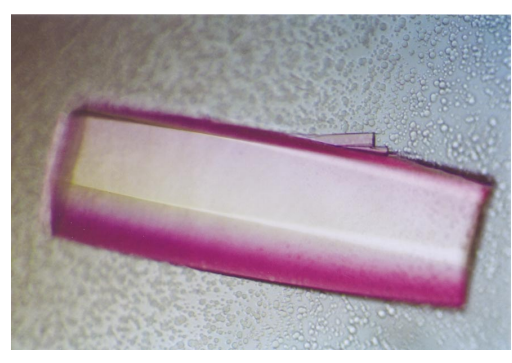

(C) 2005 International Union of Crystallography All rights reserved

\section{Four crystal forms of a Bence-Jones protein}

Four crystal forms have been grown and characterized by X-ray diffraction of a Bence-Jones protein collected from the urine of a multiple myeloma patient more than 40 years ago. Closely related tetragonal and orthorhombic forms belonging to space groups $P 4_{3} 2_{1} 2$ and $P 2_{1} 2_{1} 2_{1}$, with unit-cell parameters $a=b=68.7, c=182.1$ and $a=67.7, b=69.4, c=87.3 \AA$, diffract to 1.5 and $1.9 \AA$, respectively. Two closely related trigonal forms, both belonging to space group $P 3_{1} 21$ with unit-cell parameters $a=b=154.3 \AA$ but differing by a doubling of the $c$ axis, one $46.9 \AA$ and the other $94.0 \AA$, diffract to 2.9 and $2.6 \AA$ resolution, respectively. The trigonal crystal of short $c$-axis length shows a positive indication of twinning. The trigonal crystal of longer $c$ axis, which appeared only after eight months of incubation at room temperature, is likely to be composed of proteolytically degraded molecules and unlike the other crystal forms contains two entire Bence-Jones dimers in the asymmetric unit. This latter crystal form may shed some light on the formation of fibrils common to certain storage diseases.

\section{Introduction}

Bence-Jones proteins are monoclonal immunoglobulin light chains that are excreted in large amounts in the urine of patients having multiple myeloma, a cancer of the bone marrow (Clamp, 1967; Jones, 1847; Sanders, 1994). They are soluble molecules that also find their way into the serum of patients, where they can also become involved in deposition diseases (Solomon et al., 1982, 1991). In these latter manifestations, Bence-Jones protein that has undergone some proteolytic degradation can, in a variety of tissues, polymerize into fibrils which subsequently condense into ordered arrays. The molecular weight of a Bence-Jones $\lambda$-type light chain is $22000-24000 \mathrm{Da}$, but these invariably associate to form dimers of twice this weight (Bernier \& Putnam, 1963). The structures of a number of BenceJones proteins have been determined by X-ray crystallography (Bourne et al., 2002; Ely et al., 1989; Huang, Ainsworth, Solomon et al., 1996; Huang, Ainsworth, Stevens et al., 1996), each unique in sequence and antigen specificity and therefore in detailed features. For example, the paired constant domains and the paired variable domains of the dimer are related by an elbow angle, as are the same pairs of domains in an Fab, and this varies among Bence-Jones proteins.

The Bence-Jones protein investigated here was collected from a patient in Melbourne, Australia (initials KWR) in 1962 in near-gram quantities. It was purified shortly after collection and stored in lyophilized form for about 40 years before being crystallized by us for $\mathrm{X}$-ray analysis. The sequence of the protein has been determined by traditional protein-sequencing methods (to be published elsewhere) and the protein was found to be an immunoglobulin $\lambda$ light chain of subgroup II. Two copies of the molecule are linked covalently near the carboxy-termini through a disulfide bond to form a homodimer. In the following, we will refer to this molecule as BJ-KWR.

\section{Materials and methods}

BJ-KWR dissolved readily in $50 \mathrm{~m} M$ Tris- $\mathrm{HCl} \mathrm{pH} 8.5$ and when run on SDS-PAGE showed a single prominent band corresponding to a 
Table 1

Crystal and data-processing statistics for all crystal forms.

Values in parentheses are for the highest resolution shell.

\begin{tabular}{|c|c|c|c|c|}
\hline Space group & $P 4_{3} 2_{1} 2$ & $P 2_{1} 2_{1} 2_{1}$ & $P 3_{1} 21$ or $P 3_{2} 21$ & $P 3_{1} 21$ or $P 3_{2} 21$ \\
\hline Unit-cell parameters $(\AA)$ & $a=b=68.7, c=182.1$ & $a=67.7, b=69.4, c=87.3$ & $a=b=153.5, c=94.0$ & $a=b=155.1, c=46.9$ \\
\hline$Z$ & 8 & 4 & 12 & 6 \\
\hline$V_{\mathrm{M}}\left(\AA^{3} \mathrm{Da}^{-1}\right)$ & 2.37 & 2.26 & 3.52 & 3.59 \\
\hline Resolution (Á) & $46.94-1.49(1.54-1.49)$ & $43.65-1.90(1.97-1.90)$ & $100.0-2.9(3.0-2.9)$ & $44.79-2.60(2.69-2.60)$ \\
\hline Observations & 1307653 & 190685 & 465042 & 314311 \\
\hline Unique reflections & 57396 & 24373 & 27674 & 20152 \\
\hline Completeness (\%) & $79.1(18.2)$ & $73.9(10.6)$ & $93.4(64.2)$ & $100.0(100.0)$ \\
\hline$R_{\text {merge }}(\%)$ & $6.9(41.7)$ & $10.4(37.3)$ & $10.0(47.4)$ & $15.0(40.0)$ \\
\hline$\langle I / \sigma(I)\rangle$ & $27.1(2.4)$ & $11.9(2.2)$ & - & $11.9(3.8)$ \\
\hline$\langle I\rangle /\langle\sigma(I)\rangle$ & - & - & $14.7(2.3)$ & - \\
\hline Data-processing program used & $d^{*} T R E K$ & $d^{*} T R E K$ & $H K L 2000$ & $d^{*} T R E K$ \\
\hline
\end{tabular}

molecular weight of $29 \mathrm{kDa}$ and $51 \mathrm{kDa}$ in the presence and absence of $\beta$-mercaptoethanol, respectively. These are higher than expected from the amino-acid sequence, hence BJ-KWR must for some reason run anomalously in polyacrylamide gel. Some material of higher molecular weight was also present, which was likely to be aggregates of BJ-KWR, but there was no evidence that any degradation had occurred. The 40-year-old protein was intact. BJ-KWR was further purified by size-exclusion chromatography on Sephadex G-75, which removed a small fraction of higher molecular weight, most probably aggregates that were not completely solubilized. Mass spectrometry of the original sample, however, indicated it to be homogeneous and to have a molecular weight of $45379 \mathrm{Da}$. This further confirmed that the protein was not degraded and also that it was not glycosylated as are some Bence-Jones proteins.

Conventional vapor diffusion using Cryschem sitting-drop plates and commercially available crystallization screening kits (Hampton Research, Laguna Niguel, USA) was initially employed at 295 and $277 \mathrm{~K}$. The stock protein concentration was $23.2 \mathrm{mg} \mathrm{ml}^{-1}$ and drop sizes were $8 \mu \mathrm{l}$. Conditions yielding crystals were obtained that employed ammonium sulfate as precipitant at $295 \mathrm{~K}$. After optimization, conditions consisted of $10 \mu \mathrm{l}$ droplets containing a 1:1 ratio of

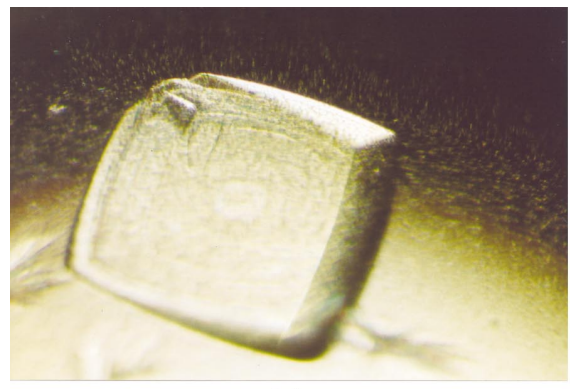

(a)

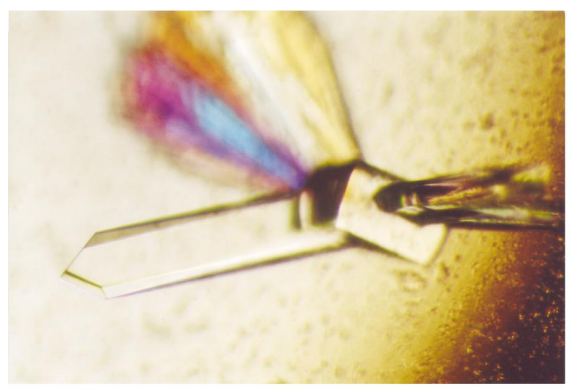

(c)

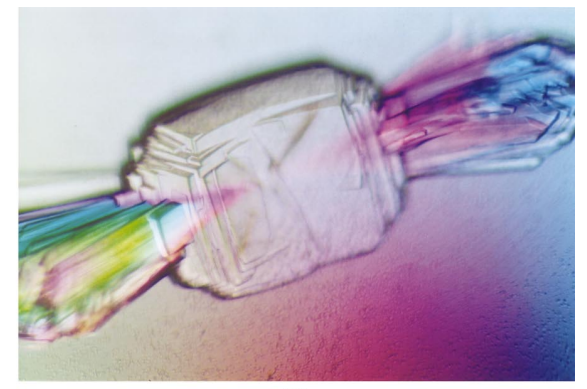

(b)

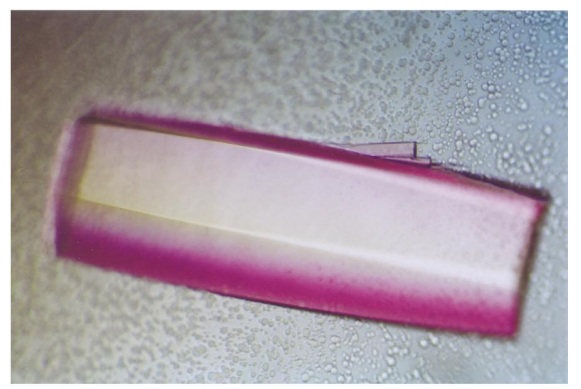

$(d)$
Figure 1

BJ-KWR protein crystals grown at $295 \mathrm{~K}$ in $(a) 1.5 \mathrm{M}$ ammonium sulfate $\mathrm{pH} 8.0,(b) 1.45 \mathrm{M}$ ammonium sulfate $\mathrm{pH} 8.5$, (c) $1.45 \mathrm{M}$ ammonium sulfate $\mathrm{pH} 8.0,(d) 2.88 M \mathrm{NaH}_{2} \mathrm{PO}_{4} / 0.72 M \mathrm{~K}_{2} \mathrm{HPO}_{4} \mathrm{pH} 4.0$.
$8.8-9.5 \mathrm{mg} \mathrm{ml}^{-1}$ protein and reservoir solution. The latter consisted of 1.4-1.6 $\mathrm{M}\left(\mathrm{NH}_{4}\right)_{2} \mathrm{SO}_{4}$ in $0.1 \mathrm{M}$ Tris- $\mathrm{HCl} \mathrm{pH} 7.0-8.5$. Using the same methodology, crystals were also grown from 2.88-3.20 M $\mathrm{NaH}_{2} \mathrm{PO}_{4}$ and $0.72-0.80 M \mathrm{~K}_{2} \mathrm{HPO}_{4}$ with $0.1 M$ buffer in the $\mathrm{pH}$ range 3.8-4.20. The buffer was constituted of $1.0 M \mathrm{Na}_{2} \mathrm{HPO}_{4}$ and $1.0 \mathrm{M}$ citric acid.

For all crystal forms investigated, representative crystals were first conventionally mounted in quartz capillaries and analyzed at $291 \mathrm{~K}$ on a laboratory X-ray diffraction apparatus. This was an R-AXIS IV system composed of a Rigaku RU-200 X-ray generator fitted with Osmic mirrors and dual image-plate detectors (Molecular Structures Corporation, Woodlands, TX, USA). For data collection to high resolution, crystals were treated with cryogens and frozen directly in a cryostream. Data were collected on beamline 5.0.2 at the Advanced Light Source at the Lawrence Berkeley National Laboratory (ALS) using $1.000 \AA$ radiation and also on beamline 7-1 at the Stanford Synchrotron Radiation Laboratory (SSRL) using $1.080 \AA$ radiation. Exposure times were $15 \mathrm{~s}$ for $0.5^{\circ}$ (tetragonal crystal) and $1.5^{\circ}$ (orthorhombic and short $c$-axis trigonal crystals) oscillation angles at ALS and $60 \mathrm{~s}$ for $1.0^{\circ}$ oscillation angles (long $c$-axis trigonal crystal) at SSRL. Data were processed and reduced using either HKL2000 (Otwinowski \& Minor, 1997) or $d^{*} T R E K$ (Pflugrath, 1999). Relevant statistics for these data are shown in Table 1.

\section{Results}

Initially, crystals of a consistent though suspicious habit were observed. The crystals, similar to that seen in Fig. 1(a), were roughly cube-shaped and often grew to $0.2 \mathrm{~mm}$ or more in edge dimension. An unsettling feature of the crystals was that their faces tended to be curved and the edges rather indistinct in some cases. However, they were physically robust and easy to mount for analysis. Later, we began to observe a second crystal habit that formed and coexisted for some time with the first and then grew, apparently at the expense of the initial form. Frequently, this secondary crystal form nucleated on and grew from the surfaces of the cube-shaped crystals, suggesting a possible unit-cell relationship (Figs. $1 b$ and $1 c$ ). Occasionally, the prismatic form appeared directly without accompaniment by the first form. 
Numerous attempts to collect diffraction data from crystals of the cube habit with rounded faces were unsuccessful. These were carried out using a home source at $295 \mathrm{~K}$ with crystals conventionally mounted in quartz capillaries. Diffraction patterns generally extended no further than $3.5 \AA$ resolution and often some zones clearly showed the presence of multiple misaligned reciprocal lattices. Nonetheless, it was sometimes possible to index these patterns and the unit cell was found to belong to space group $P 4_{1} 22$ (or $P 4_{3} 22$ ), with unit-cell parameters $a=b=64.2, c=179.7 \AA$. These crystals were the subject of a detailed atomic force microscopy (AFM) study (Plomp et al., 2003), which demonstrated among other things that the unique axis was $4_{3}$.

Eventually, an unusually large crystal of the cube habit $0.7 \mathrm{~mm}$ on an edge was obtained (Fig. 1a) and X-ray diffraction data which showed no evidence of disorder were collected at ALS to $1.5 \AA$ resolution. The data were collected from the single crystal after passing it through a series of mother-liquor solutions lacking protein containing 5, 10, 15, 20 and finally $25 \%$ glycerol. The resident time at each increment was about $10 \mathrm{~min}$ in the first three steps, $30 \mathrm{~min}$ in the fourth step and a quick wash in the $25 \%$ glycerol step. The space group of the crystal was $P 4_{3} 22_{1} 2$, with unit-cell parameters $a=b=68.7$, $c=182.1 \AA$. In contrast to previous tetragonal crystals, whose diffraction patterns had shown indications of disorder, a twinning test (Yeates, 1997) using CNS v.1.1 (Brünger et al., 1998) on this data indicated no signs of merohedral twinning. The untwinned tetragonal crystal has a volume-to-protein weight ratio $V_{\mathrm{M}}$ of $2.37 \AA^{3} \mathrm{Da}^{-1}$ (Matthews, 1968) if a single BJ-KWR dimer of $45379 \mathrm{Da}$ is assumed to be present in the asymmetric unit.

Data from crystals exposed to a similar cryopreservation procedure as for the tetragonal crystal and using the collection parameters described above were also obtained at ALS for the orthorhombic crystals seen in Fig. 1(c). Reflections were recorded to $1.9 \AA$, again from a single crystal. The space group is $P 2_{1} 2_{1} 2_{1}$, with orthorhombic unit-cell parameters $a=67.7, b=69.4, c=87.3 \AA$. The similarity in the unit-cell parameters for the tetragonal and orthorhombic crystals explains why the latter nucleated with such facility upon the surfaces of the former. For the orthorhombic crystals, $V_{\mathrm{M}}$ was $2.26 \AA^{3} \mathrm{Da}^{-1}$ and there is again one BJ-KWR dimer per asymmetric unit.

A third crystal form of BJ-KWR was also obtained and this was in many ways the most interesting. Crystallization plates which had not yielded any crystals, although conditions were basically the same as for those that had, were gathered together and stored in a dark closed cabinet at room temperature for eight months. Before disposal, the plates were examined under a microscope one last time. One, and only one, droplet out of the perhaps 3000 droplets reviewed contained six to eight beautifully formed hexagonal prisms, some having maximum lengths of nearly $0.4 \mathrm{~mm}$.

After passage through a cryopreservative consisting of 50\% MPD plus $20 \%$ PEG 3350 mixed in a 2:1 ratio with the reservoir of the droplet, crystals were frozen in the cryostream. Data were collected at SSRL beamline 7-1 to the limit of resolution, which was $2.9 \AA$. Although limited in extent, the patterns were sharp, with low mosaicity, and showed no evidence of twinning or disorder. The crystals belong to space group $P 3_{1} 21$ or $P 3_{2} 21$, with unit-cell parameters $a=b=153.5, c=94.0 \AA$. For these crystals, $V_{\mathrm{M}}$ is $3.52 \AA^{3} \mathrm{Da}^{-1}$ if one assumes two entire BJ-KWR dimers as the asymmetric unit and indeed this appears most reasonable.

Given the mysterious appearance of the trigonal crystals, we attempted to reproduce them by treating the protein with a variety of proteases and by seeking alternative crystallization conditions. Recently, trigonal crystals of similar if not identical habit were obtained at room temperature at elevated phosphate concentrations instead of ammonium sulfate (Fig. 1d). Based initially on Wizard Screen II condition No. 19 (Emerald BioStructures, Inc.), BJ-KWR trigonal crystals grew from a $1: 1$ ratio of $9.5 \mathrm{mg} \mathrm{ml}^{-1}$ protein and reservoir solution containing $2.88 M \mathrm{NaH}_{2} \mathrm{PO}_{4}$ and $0.72 M \mathrm{~K}_{2} \mathrm{HPO}_{4}$ as precipitant and $0.1 \mathrm{M} \mathrm{Na}_{2} \mathrm{HPO}_{4} /$ citric acid $\mathrm{pH} 4.0$ at $295 \mathrm{~K}$. These crystals were exposed for 2 min intervals to a simulated mother liquor minus protein solution containing increasing concentrations of ethylene glycol up to $20 \%$. The hexagonal prisms, some of which were greater than $1.2 \mathrm{~mm}$ in length, diffracted synchrotron radiation at ALS beamline 5.0.2 to 2.6 $\AA$. They had $a$ - and $b$-axis lengths virtually the same as the previous trigonal crystals. However, the $c$ axis was exactly half the length of the first trigonal crystal $c$ axis. The $V_{M}$ value would therefore be the same as the first trigonal crystal, but with only a single BJ-KWR dimer as the asymmetric unit.

The trigonal crystals obtained from phosphate-containing mother liquors appeared to be attractive candidates for structure solution. They grew readily to large size and diffracted X-rays strongly to relatively high resolution. In addition, they have only a single BJ-KWR dimer as their asymmetric unit. However, tests of the diffraction data for the presence of twinning using $C N S$ are positive. Thus, these trigonal crystals may, like the majority of the tetragonal crystals, be merohedrally twinned. Resolution of this question must await structure solution.

Currently, we are attempting to solve the structures of all four crystal forms using molecular-replacement techniques. A variety of probes based on known Bence-Jones protein structures are being investigated. Bence-Jones proteins, like Fab fragments of antibodies, may have quite variable elbow angles and there are infinite sets of CDR-loop compositions and conformations.

\section{Discussion}

BJ-KWR protein, despite spending more than 40 years as a lyophilized powder, is intact. This is amply demonstrated by SDS-PAGE and mass spectrometry. It apparently maintains a native homogenous conformation and crystallizes well under defined conditions. It shows a high propensity to form tetragonal crystals, but these are frequently twinned. However, untwinned crystals were grown and these diffracted to high resolution. This was true for the tetragonal crystal form $\left(P_{3} 2_{1} 2\right)$ as well as the closely related orthorhombic form $\left(P 2_{1} 2_{1} 2_{1}\right)$. Why this twinning occurs some, but not all of the time is unclear.

The trigonal crystals are most interesting because they appeared only after storage at room temperature for eight months. As has been pointed out (McPherson, 1999; McPherson et al., 2004), this is an almost sure sign that proteolysis has occurred and that the crystals are composed of some degraded though generally uniform version of the molecule. Unfortunately, because of the irreproducibility of these crystals and the need to commit them to data collection, no biochemical measure of the degree of degradation was obtained. The asymmetric unit of these crystals is not one BJ-KWR dimer as in the other crystal forms, but two molecules organized in pairs around a threefold screw axis. This may be significant, as it has been shown for other Bence-Jones proteins that proteolylic activity sometimes leads to fibril formation by the degradation product about some helical axis (Epstein et al., 1974; Glenner et al., 1971; Harris et al., 2000; Linke, Tischendorf et al., 1973; Linke, Zucker-Franklin et al., 1973; Solomon \& McLaughlin, 1969; Solomon et al., 1998; Wall et al., 1999). Thus, the structure of the trigonal crystals may shed some light on the physiological assembly and structure of Bence-Jones protein that occurs in primary amyloidosis and other storage diseases. 
This work was supported by $\mathrm{CNPq}$ (Conselho Nacional de Desenvolvimento Científico e Tecnológico), an entity of the Brazilian government for the development of science and technology, and by grants from NASA and the NIH (GM58868-02).

\section{References}

Bernier, G. M. \& Putnam, F. W. (1963). Nature (London), 200, 223-225.

Bourne, P. C., Ramsland, P. A., Shan, L., Fan, Z. C., DeWitt, C. R., Shultz, B. B., Terzyan, S. S., Moomaw, C. R., Slaughter, C. A., Guddat, L. W. \& Edmundson, A. B. (2002). Acta Cryst. D58, 815-823.

Brünger, A. T., Adams, P. D., Clore, G. M., DeLano, W. L., Gros, P., GrosseKunstleve, R. W., Jiang, J.-S., Kuszewski, J., Nilges, M., Pannu, N. S., Read, R. J., Rice, L. M., Simonson, T. \& Warren, G. L. (1998). Acta Cryst. D54, 905-921.

Clamp, J. R. (1967). Lancet, 290, 1317-1374.

Ely, K. R., Herron, J. N., Harker, M. \& Edmundson, A. B. (1989). J. Mol. Biol. 210, 601-615.

Epstein, W. V., Tan, M. \& Wood, I. S. (1974). J. Lab. Clin. Med. 84, 107-110.

Glenner, G. G., Ein, D., Eanes, E. D., Bladen, H. A., Terry, W. \& Page, D. L. (1971). Science, 174, 712-714.

Harris, D. L., King, E., Ramsland, P. A. \& Edmundson, A. B. (2000). J. Mol. Recognit. 13, 198-212.

Huang, D. B., Ainsworth, C., Solomon, A. \& Schiffer, M. (1996). Acta Cryst. D52, 1058-1066.
Huang, D. B., Ainsworth, C. F., Stevens, F. J. \& Schiffer, M. (1996). Proc. Natl Acad. Sci. USA, 93, 7017-7021.

Jones, B. H. (1847). Lancet, 50, 88-92.

Linke, R. P., Tischendorf, F. W., Zucker-Franklin, D. \& Franklin, E. C. (1973). J. Immunol. 111, 24-26.

Linke, R. P., Zucker-Franklin, D. \& Franklin, E. C. (1973). J. Immunol. 111, 10 23.

McPherson, A. (1999). Crystallization of Biological Macromolecules, pp. 586. Cold Spring Harbor, USA: Cold Spring Harbor Laboratory Press.

McPherson, A., Day, J. \& Harris, L. J. (2004). Acta Cryst. D60, 872877.

Matthews, B. W. (1968). J. Mol. Biol. 33, 491-497.

Otwinowski, Z. \& Minor, W. (1997). Methods Enzymol. 276, 307-326.

Pflugrath, J. W. (1999). Acta Cryst. D55, 1718-1725.

Plomp, M., McPherson, A. \& Malkin, A. J. (2003). Proteins, 50, 486-495.

Sanders, P. W. (1994). J. Lab. Clin. Med. 124, 484-488.

Solomon, A., Frangione, B. \& Franklin, E. C. (1982). J. Clin. Invest. 70, 453460.

Solomon, A. \& McLaughlin, C. L. (1969). J. Biol. Chem. 244, $3393-$ 3404.

Solomon, A., Weiss, D. T. \& Kattine, A. A. (1991). N. Engl. J. Med. 324, 1845 1851.

Solomon, A., Weiss, D. T., Murphy, C. L., Hrncic, R., Wall, J. S. \& Schell, M. (1998). Proc. Natl Acad. Sci. USA, 95, 9547-9551.

Wall, J., Murphy, C. L. \& Solomon, A. (1999). Methods Enzymol. 309, 204-217. Yeates, T. O. (1997). Methods Enzymol. 276, 344-358. 